\title{
Measuring the effective phonon density of states of a quantum dot in cavity quantum electrodynamics
}

Madsen, Kristian Høeg; Nielsen, Per Kær; Kreiner-Møller, Asger; Stobbe, Søren; Nysteen, Anders; Mørk, Jesper; Lodahl, Peter

Published in:

Physical Review B Condensed Matter

Link to article, DOI:

10.1103/PhysRevB.88.045316

Publication date:

2013

Document Version

Publisher's PDF, also known as Version of record

Link back to DTU Orbit

Citation $(A P A)$ :

Madsen, K. H., Nielsen, P. K., Kreiner-Møller, A., Stobbe, S., Nysteen, A., Mørk, J., \& Lodahl, P. (2013).

Measuring the effective phonon density of states of a quantum dot in cavity quantum electrodynamics. Physical Review B Condensed Matter, 88(4), [045316]. https://doi.org/10.1103/PhysRevB.88.045316

\section{General rights}

Copyright and moral rights for the publications made accessible in the public portal are retained by the authors and/or other copyright owners and it is a condition of accessing publications that users recognise and abide by the legal requirements associated with these rights.

- Users may download and print one copy of any publication from the public portal for the purpose of private study or research.

- You may not further distribute the material or use it for any profit-making activity or commercial gain

- You may freely distribute the URL identifying the publication in the public portal 


\title{
Measuring the effective phonon density of states of a quantum dot in cavity quantum electrodynamics
}

\author{
K. H. Madsen,,${ }^{1,2,}{ }^{*}$ P. Kaer, ${ }^{2}$ A. Kreiner-Møller, ${ }^{2}$ S. Stobbe, ${ }^{1}$ A. Nysteen, ${ }^{2}$ J. Mørk ${ }^{2}$ and P. Lodahl ${ }^{1, \dagger}$ \\ ${ }^{1}$ Niels Bohr Institute, University of Copenhagen, Blegdamsvej 17, DK-2100 Copenhagen, Denmark \\ ${ }^{2}$ DTU Fotonik, Department of Photonics Engineering, Technical University of Denmark, Ørsteds Plads 343, DK-2800 Kgs. Lyngby, Denmark
}

(Received 24 May 2012; revised manuscript received 5 July 2013; published 24 July 2013)

\begin{abstract}
We employ detuning-dependent decay-rate measurements of a quantum dot in a photonic-crystal cavity to study the influence of phonon dephasing in a solid-state quantum-electrodynamics experiment. The experimental data agree with a microscopic non-Markovian model accounting for dephasing from longitudinal acoustic phonons, and the analysis explains the difference between nonresonant cavity feeding in different nanocavities. From the comparison between experiment and theory we extract the effective phonon density of states experienced by the quantum dot in the nanocavity. This quantity determines all phonon dephasing properties of the system and is found to be described well by a theory of bulk phonons.
\end{abstract}

DOI: 10.1103/PhysRevB.88.045316

PACS number(s): 78.67.Hc, 03.65.Yz, 42.50.Pq, 63.20.kd

\section{INTRODUCTION}

Mechanical motion due to thermal vibrations, i.e., phonons, is omnipresent in solid-state systems and inevitably leads to decoherence of quantum superpositon states encoded in the system. Understanding and ultimately engineering such phonon processes may lead to new opportunities for coherent light-matter interaction in an all-solid-state environment. So far, quantitative measurements of the intrinsic phonondephasing mechanisms have not been presented, which implies that the full potential of quantum dots (QDs) for on-chip quantum-photonics applications has not yet been explored. ${ }^{1}$ For example, it has been predicted that a proper account of the phonon decoherence mechanism is required to achieve highly indistinguishable single photons from QDs in cavities. ${ }^{2}$ Furthermore, the emerging field of quantum optomechanics explores the ultimate quantum mechanical motion of, e.g., nanomembranes, ${ }^{3}$ and generating carriers in semiconductor membranes has been proven to enable novel mechanical cooling mechanisms. ${ }^{4,5}$

Cavity quantum-electrodynamics (QED) experiments in solid-state systems have yielded major breakthroughs within the last decade, including the observation of Purcell enhancement, ${ }^{6,7}$ strong coupling between a single QD and a photon, ${ }^{8,9}$ and non-Markovian dynamics. ${ }^{10}$ A number of surprises have emerged, distinguishing QD-based QED from its atomic counterpart, including the breakdown of the pointdipole description of light-matter interaction, ${ }^{11}$ the role of phonon dephasing, ${ }^{12-14}$ and multiple-charge transitions. ${ }^{15,16}$ In this paper, we explore the role of environmental fluctuations by employing a QD embedded in a nanocavity as a sensitive probe of the phonon dephasing processes in a photoniccrystal (PC) nanomembrane. We compare our experimental data to a microscopic theory for longitudinal acoustic (LA) phonons and extract the effective phonon density of states (DOS) for the QD, which is the key concept describing all aspects of phonon dephasing. Phonon sidebands have been observed ${ }^{17}$ and modeled ${ }^{18}$ in spectral measurements on QDs in homogenous structures at elevated temperatures where phonon dephasing is very pronounced. Previous work on detuning-dependent decay rates in a PC nanocavity has established the importance of phonon dephasing for the Purcell enhancement. ${ }^{12}$ In the present experiment, the dynamics of a single QD enables a direct measurement of the phonon decoherence mechanism, which is possible since the QD is coherently coupled to a cavity. Our work constitutes a significant extension, into the realm of coherent quantum optics, of previous work where proper account of the QD fine structure enabled probing the incoherent local optical density of states (LDOS) ${ }^{19}$ The applied method is expected to have widespread applications for probing phonon dephasing in advanced nanostructures where the combination of photonic and phononic band gaps ${ }^{20}$ ultimately could enable complete coherent control over single-photon emission from QDs.

\section{EXPERIMENTAL DATA}

We investigate a GaAs PC membrane with lattice constant $a=240 \mathrm{~nm}$, hole radius $r=65 \mathrm{~nm}$, and thickness $154 \mathrm{~nm}$ containing self-assembled InGaAs QDs with a density of $\sim 80 \mu \mathrm{m}^{-2}$. An L3 cavity is introduced by leaving out three holes [see Fig. 1(c) inset] and shifting the three first holes at each end of the cavity by $0.175 a, 0.025 a$, and $0.175 a$, respectively. ${ }^{21}$ We measure $Q=6690 \pm 37$ corresponding to a cavity decay rate of $\hbar \kappa=195 \pm 1 \mu \mathrm{eV}$ by saturating the QDs and recording the cavity linewidth. We employ confocal microscopy, and the collected emission is sent to an avalanche photo detector (APD) for time-resolved measurements. Whereas above-band excitation gives rise to emission from many QDs and multiexcitons within each QD, we tune the excitation laser into resonance with higher-order modes of the cavity, thus enabling selective excitation of QDs positioned within the cavity. The fundamental and high- $Q$ mode of the cavity (M1) is observed at $952 \mathrm{~nm}$, and we use the sixth cavity mode (M6) situated at $850 \mathrm{~nm}$ for excitation. ${ }^{22}$

In cavity QED, phonons in the solid-state environment can significantly alter the dynamics of the QD. ${ }^{14}$ Thus, a QD detuned many linewidths away from the cavity resonance can emit photons to the cavity with a Purcell-enhanced rate through the accompanying emission (absorption) of a phonon for positive (negative) detuning, which is defined as the frequency difference between the QD transition and the cavity mode, $\Delta=$ $\omega_{\mathrm{qd}}-\omega_{\mathrm{ca}}$. At low temperatures, a large detuning asymmetry 

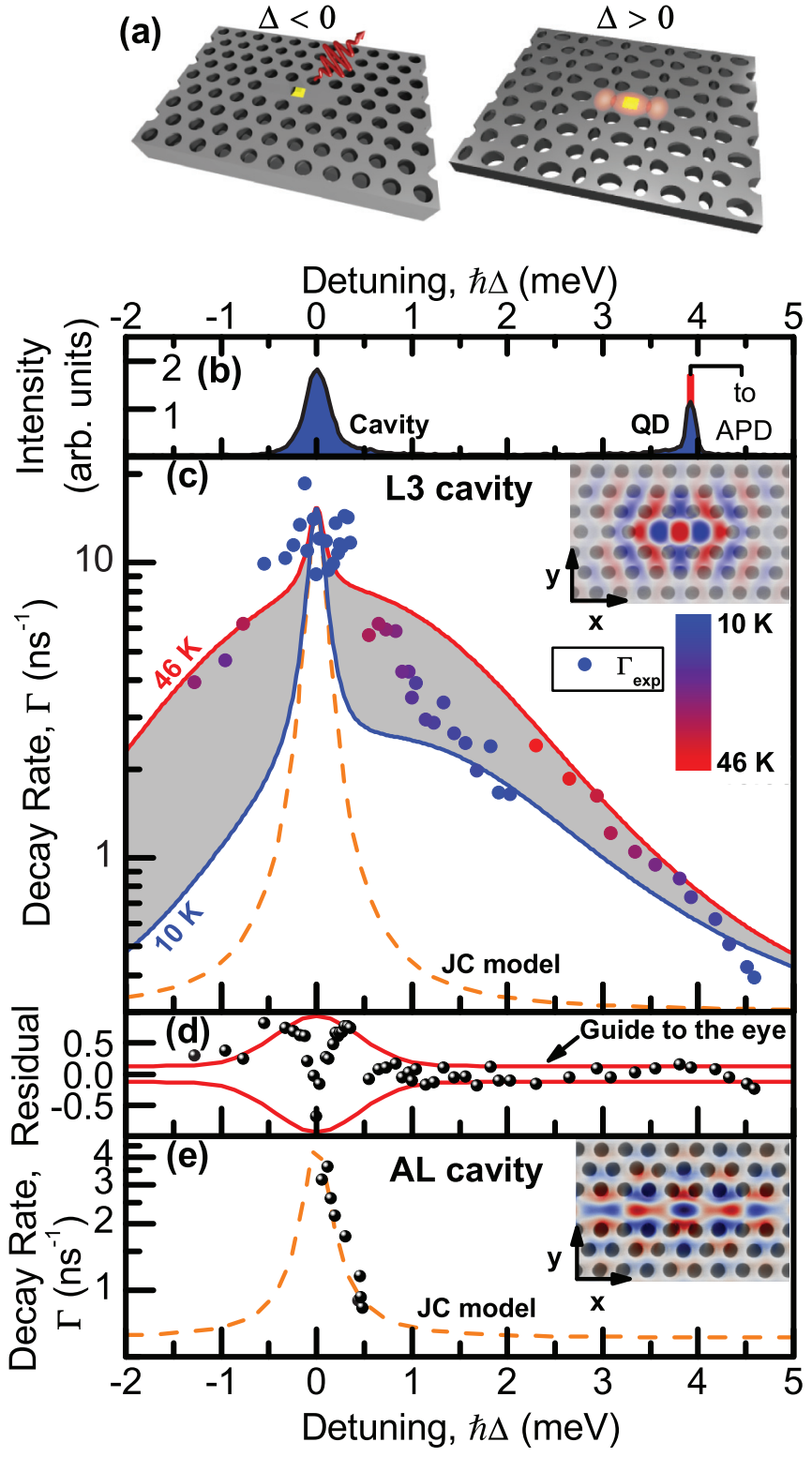

FIG. 1. (Color online) (a) Illustration of physical mechanism behind phonon-enhanced Purcell effect. For negative (positive) detuning, the QD (yellow point) can decay into the cavity by absorbing (emitting) a phonon. The emitted LA phonon distorts the lattice of holes in the PC membrane. At $T=0 \mathrm{~K}$ no phonon absorption is possible and residual spontaneous emission (red wavepacket) will dominate for negative detuning. (b) Emission spectrum with the indication of spectral range detected by the APD. (c) Measured decay rate versus detuning with the color specifying the experimental temperature. The corresponding theory is plotted for the largest and smallest temperatures applied in the experiment (solid curves), and the experimental data are found to be within these predicted bounds. The dotted orange curve is the prediction from the JC model. The applied excitation power is 1.5 times the saturation power of the QD except for $\Delta<-0.5 \mathrm{meV}$ and $\Delta>2 \mathrm{meV}$, where the power is 0.7 times the saturation power. (d) Residuals $\left(\Gamma_{\exp }-\Gamma_{\text {the }}\right) / \Gamma_{\exp }$ between experiment and theory. (e) Decay rate measurements for an Anderson-localized cavity in a disordered PC waveguide that are well explained by a JC model. Insets in (c) and (e) show SEM images of the physical systems, where the $y$ components of the simulated electric fields are overlayed. is expected since the thermal phonon occupancy is low. For example, at $T=10 \mathrm{~K}$ and phonon energies larger than $1 \mathrm{meV}$, which are typical values in the present experiment, we estimate $n \leqslant 0.45$ meaning that the probabilities for phonon emission $(\propto n+1)$ and absorption $(\propto n)$ vary significantly. The phononassisted decay is illustrated in Fig. 1(a). For positive detuning the QD can decay through the cavity and in this process creates lattice vibrations, while the corresponding absorption process is suppressed for negative detunings and the QD decays mainly by coupling to radiation modes. The phonon-assisted processes are inherently non-Markovian implying that the memory of the phononic reservoir cannot be neglected and the phonon reservoir is "colored," i.e., frequency dependent. ${ }^{14}$ As explained below, this non-Markovian frequency dependence can be probed experimentally by detuning-dependent decayrate measurements of a single QD in a cavity.

In Fig. 1(c) the QD decay rate is plotted as a function of detuning. Temperature control $(10-46 \mathrm{~K})$ and gas deposition on the sample enable redshifting the QD and cavity frequency, respectively. We observe Purcell enhancement that is spectrally much broader than expected from the standard Markovian Jaynes-Cummings (JC) model. An example of a spectral measurement is shown in Fig. 1(b). We observe the previously reported long-range coupling between QD and cavity by the strong emission from the cavity even for large detunings, ${ }^{23}$ however, the spectral domain is not well suited for extracting phonon dephasing processes of single excitons since multiple charge configurations in the QDs have been shown to feed the cavity. ${ }^{15}$ For QDs tuned out of the cavity, see Fig. 1(b), the dynamics of single exciton lines under the sole influence of phonon dephasing is considered, because only a small spectral window is sent to the APD. The decay curves are bi-exponential due to the presence of a bright and dark state, and the fast rate gives the decay of the bright state, which is the rate relevant for comparison with theory. For smaller detunings, $\hbar|\Delta|<0.5 \mathrm{meV}$, cavity feeding from additional exciton lines influences the decay curves that consequently appear to be multi-exponential, and we fit these decay curves with triple exponentials and extract the fast rate that is expected to be dominated by the resonant exciton. The linear power dependence together with the bi-exponential decay away from resonance proves that we probe a neutral exciton. In the present experiment we focus mainly on the large-detuning case where phonon-dephasing processes are reliably extracted. The statistical uncertainty of the decay rates can be judged from the point-to-point fluctuations in the data of Fig. 1(c).

\section{MODELING THE DECAY RATES}

We describe the QD-cavity coupling with a microscopic model that accounts for the solid-state environment through LA-phonon dephasing of the polariton quasiparticles formed in the cavity. Recently, photon-phonon interaction has been demonstrated in optically pumped L3 PC cavities, ${ }^{24}$ but such effects are many orders of magnitude smaller than the other coupling terms in the Hamiltonian for the situation of a single-QD photon source considered here. Memory effects of the phonon bath are taken into account, and we restrict to single phonon absorbtion and emission processes. In the weak-coupling regime the detuning-dependent decay rate of 
the QD is expressed as ${ }^{25}$

$$
\Gamma=\gamma+2 g^{2} \frac{\gamma_{\mathrm{tot}}}{\gamma_{\mathrm{tot}}^{2}+\Delta^{2}}\left[1+\frac{1}{\hbar^{2} \gamma_{\mathrm{tot}}} \Phi(\Omega=\Delta, T)\right],
$$

where $g$ is the light-matter coupling strength and $\gamma_{\text {tot }}=(\gamma+$ $\kappa) / 2$, where $\gamma$ is the decay rate associated with coupling to radiation modes and nonradiative recombination.

The effective phonon density experienced by the QD is defined as

$$
\begin{aligned}
\Phi(\Omega, T)= & \pi \sum_{k}\left|M^{k}\right|^{2}\left[n_{k}(T) \delta\left(\Omega+\Omega_{k}\right)\right. \\
& \left.+\left(n_{k}(T)+1\right) \delta\left(\Omega-\Omega_{k}\right)\right],
\end{aligned}
$$

where $\left|M^{k}\right|^{2}$ is the electron-phonon coupling strength, $n_{k}(T)=\left[\exp \left(\hbar \Omega_{k} / k_{B} T\right)-1\right]^{-1}$ is the Bose-Einstein distribution, and $\Omega_{k}$ is the frequency, all for the $k$ th phonon mode. The calculated $\Phi(\Omega=\Delta)$ versus detuning is plotted in the inset of Fig. 3 for increasing temperature. It vanishes for negative detuning at $T=0 \mathrm{~K}$, since here no phonons are available for absorption, while it is nonzero for positive detuning since the QD can spontaneously emit a phonon enabling the emission of a photon at the cavity frequency. As the temperature increases, the asymmetry gradually levels out since the imbalance between phonon absorption and emission processes disappears. For the experimental decay rate $\Gamma_{\exp }$ we also calculated the decay rate predicted from theory $\Gamma_{\text {the }}$ at the temperature of the particular experiment. The excellent agreement between experiment and theory for detunings $\hbar|\Delta|>0.5 \mathrm{meV}$ where the single QD line can be resolved is illustrated by the residuals plotted in Fig. 1(d). Equation (1) constitutes a direct link between the effective phonon density and the QD decay rate. This reveals the interesting insight that by embedding a QD in a cavity the detailed information about dephasing can be extracted from the dynamics of the QD population. The efficient coupling of the QD to the cavity strongly amplifies the weak phonon effects thereby making them measurable, and in the absence of the cavity coherent effects are only revealed from the QD polarization. Indeed, increasing the light-matter coupling strength (i.e., $g$ ) implies that the term containing the phonon density increases relative to the background decay rate $\gamma$, thereby making it visible, as described by Eq. (1).

\section{COMPARISON TO OTHER NANOCAVITIES}

The broadband Purcell enhancement in Fig. 1(c) was not observed in previous work on micropillar cavities, ${ }^{10}$ and in order to explain and exploit the origin of these differences we have experimentally studied yet another type of solid-state cavity system, namely an Anderson-localized (AL) cavity formed in a PC waveguide with scattering imperfections. The sample is displayed in the inset of Fig. 1(e). Here, random cavities are generated by randomly perturbing the hole positions in the three rows of holes on each side of the waveguide with a standard deviation of $3 \%$ of the lattice parameter $a$. Cavity QED with this sample was previously reported. ${ }^{26}$ In the AL cavity we observe no broadband Purcell enhancement, and the detuning dependence is well described

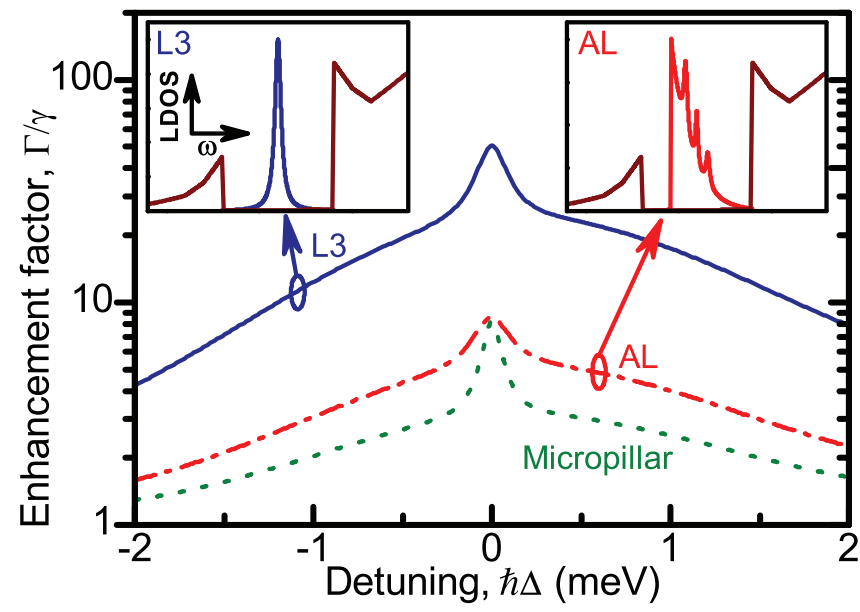

FIG. 2. (Color online) Calculation of the detuning dependence of the enhancement factor for three different cavity QED systems: the L3 PC cavity, an AL cavity, and a micropillar cavity at $T=30 \mathrm{~K}$. For the first two systems the parameters extracted from the present experiment are used while the latter corresponds to the experiment of Ref. 10. Insets show sketches of the optical LDOS for the L3 cavity and AL cavity.

by the Markovian JC model, ${ }^{27}$ cf. Fig. 1(e), despite the fact that the measured coupling strength and $Q$ factor of the AL and L3 cavities do not differ significantly. Thus we find $\hbar \kappa_{\mathrm{AL}}=230 \pm 12 \mu \mathrm{eV}(Q=5700 \pm 288)$ and $\hbar g_{\mathrm{AL}}=$ $13.3 \mu \mathrm{eV}$ for the AL cavity, which should be compared to $\hbar \kappa=195 \pm 1 \mu \mathrm{eV}$ and $\hbar g=22 \pm 0.7 \mu \mathrm{eV}$ for the L 3 cavity. As we demonstrate below, a crucial difference between the two cavities stems from the different background decay rates that are obtained from far-detuned decay rates. We record $\hbar \gamma=0.2 \mu \mathrm{eV}$ for the $\mathrm{L} 3$ cavity and $\hbar \gamma_{\mathrm{AL}}=0.4 \mu \mathrm{eV}$ for the AL cavity.

The difference between AL and micropillar cavities on the one hand and L3 PC cavities on the other can be explained from Eq. (1). In Fig. 2 we show the Purcell enhancement factor $\Gamma / \gamma$ for three different cavities calculated using Eq. (1) with identical effective phonon densities and all additional parameters determined from experimental data. We find that $\gamma$ plays a decisive role in determining the visibility of the phonon influence on the decay dynamics, and it varies significantly for the different cavity geometries. The insets in Fig. 2 show a sketch of the LDOS for the L3 and AL cavities, highlighting that while L3 PC cavities appear in the band gap where the background decay rate is strongly suppressed, ${ }^{19} \mathrm{AL}$ cavities appear as random resonances on top of a background LDOS representing the waveguide mode ${ }^{26}$ giving rise to an emission channel. Similarly in micropillar cavities the coupling to radiation modes is not strongly suppressed. In more quantitative terms two requirements need to be fulfilled in order to see broadband Purcell enhancement: $\frac{\Phi(\Delta)}{\hbar^{2} \gamma_{\text {tot }}} \geqslant 1$ and $\frac{2 g^{2}}{\hbar^{2} \gamma} \frac{\Phi(\Delta)}{\Delta^{2}} \geqslant 1$. The former weak condition is for a cavity with $Q=6690$ fulfilled for detunings above $0.45 \mathrm{meV}$. The latter and stronger condition can be evaluated to $\frac{2 g^{2}}{\gamma} \times(0.6 \mathrm{ps})=4.47 \geqslant 1$ for a typical detuning of $2 \mathrm{meV}$. This value is 5.5 times larger for the $\mathrm{L} 3$ compared to the AL cavity. 


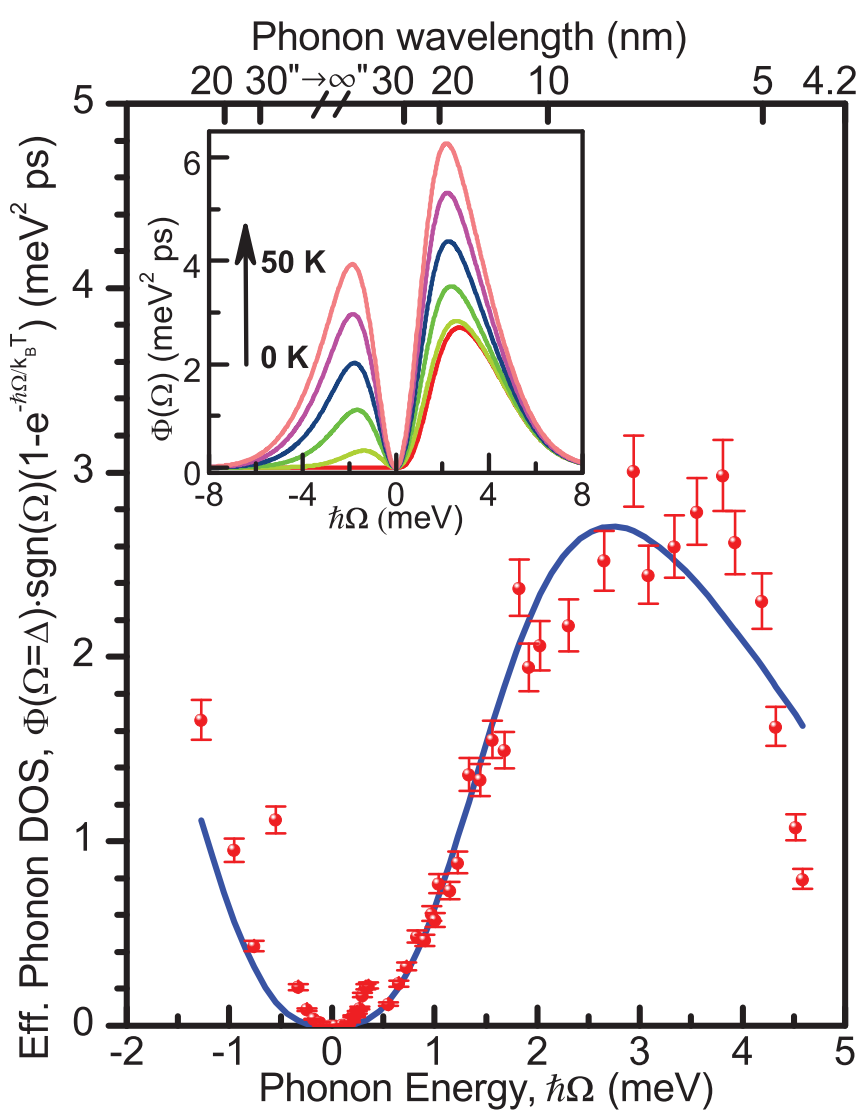

FIG. 3. (Color online) Effective phonon DOS for a QD versus phonon energy extracted from the data in Fig. 1(c). The upper axis indicates the corresponding phonon wavelength. At large phonon energies (short wavelengths) a cutoff is observed where the DOS drops. The blue curve shows the corresponding theory assuming LA phonons in a bulk GaAs. The inset shows the role of temperature on the effective phonon density.

\section{EXTRACTING AND MODELING THE EFFECTIVE PHONON DENSITY OF STATES}

Figure 3 shows the measured effective phonon DOS extracted from the data in Fig. 1(c). In order to compensate for the effect of temperature through the phonon occupation, we plot the temperature-independent quantity $\Phi(\Omega=\Delta) \operatorname{sgn}(\Delta)(1-$ $e^{-\hbar \Omega / k_{\mathrm{B}} T}$ ), which is the effective phonon DOS experienced by the QD. This corresponds to dividing by $n(T)$ and $(n(T)+1)$ for negative and positive detunings respectively, which cancels the temperature dependence in Eq. (2). The observed energy dependence of the effective phonon DOS is a direct signature of the non-Markovianity of the phonon reservoir. The phonon DOS is low for small phonon energies and grows rapidly with energy, reaching a maximum at about $3 \mathrm{meV}$. This energy cutoff originates from the phonon wavelength (of about $7 \mathrm{~nm}$ ) becoming comparable in size to the wave function of the exciton confined in the QD. ${ }^{28}$ Thus, tailoring the QD size may be employed for influencing phonon-dephasing processes. We stress that the applied method for extracting the phonon DOS is general and may be exploited in the future in more advanced nanostructures combining photonic and phononic band gaps, thus potentially enabling complete coherent control over the single-photon emission from the QDs, which will be very valuable for all-solid-state quantum information processing.

We finally compare our experimental data of the effective phonon DOS to calculations assuming bulk phonons in GaAs, cf. Fig. 3. Very good agreement between experiment and theory is observed, in particular for energies larger than $0.5 \mathrm{meV}$ where the effects of multiexciton processes or other QDs are negligible. The remarkable success of the bulk-phonon theory excludes effects of localization of phonon modes in the cavity. Phonon localization in an L3 PC cavity has been reported at energies up to $4.1 \mu \mathrm{eV},{ }^{24}$ while here typical phonon energies required for the photon-assisted recombination are above $0.5 \mathrm{meV}$. The corresponding phonon wavelength is below $42 \mathrm{~nm}$, which is much smaller than the dimensions of the cavity, explaining the success of the bulk-phonon theory. We note that localized phonons could play a role for the minor deviations from theory observed at small phonon energies. In the theory, we expand the bulk phonons in plane waves, and both the excited and ground state electron wave functions in the QD are assumed to be Gaussian in all three dimensions and circular symmetric in the $x y$ plane orthogonal to the growth direction, and they can be written

$$
\psi_{\nu}(\mathbf{r})=\frac{1}{\pi^{3 / 4} l_{v, x y} l_{\nu, z}^{1 / 2}} e^{-\left(x^{2}+y^{2}\right) / 2 l_{v, x y}^{2}} e^{-z^{2} / 2 l_{v, z}^{2}},
$$

where $v=\{\mathrm{e}, \mathrm{g}\}$ and $l_{v, x y}$ and $l_{v, z}$ determine the widths. The subscripts e and $g$ denote the excited and ground states of the electron. This allows us to express the effective phonon density as

$$
\begin{aligned}
& \Phi(\Omega, T)=\frac{\hbar}{4 \pi d c_{l}^{5}} \frac{\Omega^{3}}{1-e^{-\hbar \Omega / k_{\mathrm{B}} T}} \int_{0}^{1} d u\left(D_{\mathrm{e}} e^{\tilde{\Omega}_{e, x y}^{2}\left(u^{2}-1\right)-\tilde{\Omega}_{e, z}^{2} u^{2}}\right. \\
& \left.-D_{\mathrm{g}} e^{\tilde{\Omega}_{g, x y}^{2}\left(u^{2}-1\right)-\tilde{\Omega}_{g, z}^{2} u^{2}}\right)^{2},
\end{aligned}
$$

where $\tilde{\Omega}_{v, \mu}=\frac{\Omega l_{v, \mu}}{2 c_{l}}$ and $\mu=\{\mathrm{xy}, \mathrm{z}\} . D_{\mathrm{e}}=-14.6 \mathrm{eV}$ and $D_{\mathrm{g}}=-4.8 \mathrm{eV}$ are the deformation potentials, $c_{l}=5110 \mathrm{~m} / \mathrm{s}$ is the longitudinal speed of sound averaged over the three different crystal axes, and $d=5370 \mathrm{~kg} / \mathrm{m}^{3}$ is the density of GaAs. ${ }^{29}$ We successfully model the data with the only restriction that the height is smaller than the width, i.e., $l_{\mathrm{xy}}>l_{\mathrm{z}}$, and we obtain the following realistic widths of the electron wave functions: $l_{\mathrm{e}, \mathrm{xy}}=3.4 \mathrm{~nm}, l_{\mathrm{e}, \mathrm{z}}=1.4 \mathrm{~nm}, l_{\mathrm{g}, \mathrm{xy}}=3.9 \mathrm{~nm}$, and $l_{\mathrm{g}, \mathrm{z}}=2.3 \mathrm{~nm}$. While these sizes are used as free parameters in the model, an important consistency check is to determine the QD oscillator strength of 15.5, in very good agreement with previous measurements. ${ }^{30,31}$ Remarkably, the only additional free parameter is an overall scaling factor of the curve of 5.56, which corresponds to dividing the speed of sound by $1.41 \mathrm{in} \mathrm{Eq.} \mathrm{(4).} \mathrm{This} \mathrm{is} \mathrm{partly} \mathrm{attributed} \mathrm{to} \mathrm{the} \mathrm{anisotropy} \mathrm{of}$ sound velocity in GaAs, which is not explicitly accounted for in the theory but the experimental uncertainty in, e.g., the deformation potentials may also contribute. We stress the importance of accounting for the microscopic non-Markovian dephasing processes: for large detunings the quantity $\Phi(\Delta \gg 0) \hbar^{-2}$ enters in the theory as an effective puredephasing rate, but it is typically three orders of magnitude larger than dephasing rates extracted when interpreting experiments with a Markovian model. ${ }^{32}$ 


\section{CONCLUSION}

In conclusion, we have observed broadband Purcell enhancement for a QD in a PC cavity. Our data are very well explained by a microscopic non-Markovian LA phonon theory. We record for the first time the effective phonon DOS of a QD by employing a PC nanocavity to increase sensitivity. Remarkably, our measurements are well described by a bulk phonon theory despite the inhomogeneity of PC cavities. This work is an essential step towards understanding and potentially controlling and engineering the coherence properties of QD- based QED systems, which is required when applying such an all-solid-state platform for quantum-information processing.

\section{ACKNOWLEDGMENTS}

We thank H. Thyrrestrup and P. D. García for providing the simulated field profiles. We gratefully acknowledge financial support from the Villum Foundation, the Danish Council for Independent Research, (Natural Sciences and Technology and Production Sciences) and the European Research Council (ERC consolidator grant“ALLQUANTUM”). *khmadsen@nbi.ku.dk

†lodahl@nbi.ku.dk; www.quantum-photonics.dk

${ }^{1}$ J. L. O'Brien, A. Furusawa, and J. Vuckovic, Nat. Photon. 3, 687 (2009).

${ }^{2}$ P. Kaer, P. Lodahl, A.-P. Jauho, and J. Mørk, Phys. Rev. B 87, 081308(R) (2013).

${ }^{3}$ T. J. Kippenberg and K. J. Vahala, Science 321, 1172 (2008).

${ }^{4}$ I. Wilson-Rae, P. Zoller, and A. Imamoglu, Phys. Rev. Lett. 92, 075507 (2004).

${ }^{5}$ K. Usami, A. Naesby, T. Bagci, B. Melholt Nielsen, J. Liu, S. Stobbe, P. Lodahl, and E. S. Polzik, Nat. Phys. 8, 168 (2012).

${ }^{6}$ J. M. Gérard, B. Sermage, B. Gayral, B. Legrand, E. Costard, and V. Thierry-Mieg, Phys. Rev. Lett. 81, 1110 (1998).

${ }^{7}$ D. Englund, D. Fattal, E. Waks, G. Solomon, B. Zhang, T. Nakaoka, Y. Arakawa, Y. Yamamoto, and J. Vuckovic, Phys. Rev. Lett. 95, 013904 (2005).

${ }^{8}$ T. Yoshie, A. Scherer, J. Hendrickson, G. Khitrova, H. M. Gibbs, G. Rupper, C. Ell, O. B. Shchekin, and D. G. Deppe, Nature (London) 432, 200 (2004).

${ }^{9}$ K. Hennessy, A. Badolato, M. Winger, D. Gerace, M. Atatüre, S. Gulde, S. Fält, E. L. Hu, and A. Imamoglu, Nature (London) 445, 896 (2007).

${ }^{10}$ K. H. Madsen, S. Ates, T. Lund-Hansen, A. Löffler, S. Reitzenstein, A. Forchel, and P. Lodahl, Phys. Rev. Lett. 106, 233601 (2011).

${ }^{11}$ M. L. Andersen, S. Stobbe, A. S. Sørensen, and P. Lodahl, Nat. Phys. 7, 215 (2011).

${ }^{12}$ U. Hohenester, A. Laucht, M. Kaniber, N. Hauke, A. Neumann, A. Mohtashami, M. Seliger, M. Bichler, and J. J. Finley, Phys. Rev. B 80, 201311 (2009).

${ }^{13}$ M. Calic, P. Gallo, M. Felici, K. A. Atlasov, B. Dwir, A. Rudra, G. Biasiol, L. Sorba, G. Tarel, V. Savona, and E. Kapon, Phys. Rev. Lett. 106, 227402 (2011).

${ }^{14}$ P. Kaer, T. R. Nielsen, P. Lodahl, A.-P. Jauho, and J. Mørk, Phys. Rev. Lett. 104, 157401 (2010).

${ }^{15}$ M. Winger, T. Volz, G. Tarel, S. Portolan, A. Badolato, K. J. Hennessy, E. L. Hu, A. Beveratos, J. Finley, V. Savona, and A. Imamoglu, Phys. Rev. Lett. 103, 207403 (2009).

${ }^{16}$ N. Chauvin, C. Zinoni, M. Francardi, A. Gerardino, L. Balet, B. Alloing, L. H. Li, and A. Fiore, Phys. Rev. B 80, 241306 (2009).
${ }^{17}$ L. Besombes, K. Kheng, L. Marsal, and H. Mariette, Phys. Rev. B 63, 155307 (2001).

${ }^{18}$ F. Rol, S. Founta, H. Mariette, B. Daudin, L. S. Dang, J. Bleuse, D. Peyrade, J.-M. Gérard, and B. Gayral, Phys. Rev. B 75, 125306 (2007).

${ }^{19}$ Q. Wang, S. Stobbe, and P. Lodahl, Phys. Rev. Lett. 107, 167404 (2011).

${ }^{20}$ M. Eichenfield, J. Chan, R. M. Camacho, K. J. Vahala, and O. Painter, Nature (London) 462, 78 (2009).

${ }^{21}$ Y. Akahane, T. Asano, B.-S. Song, and S. Noda, Opt. Express 13, 1202 (2005).

${ }^{22}$ M. Kaniber, A. Neumann, A. Laucht, M. F. Huck, M. Bichler, M.-C. Amann, and J. J. Finley, New J. Phys. 11, 013031 (2009).

${ }^{23}$ S. Ates, S. M. Ulrich, A. Ulhaq, S. Reitzenstein, A. Löffler, S. Höfling, A. Forchel, and P. Michler, Nat. Photon. 3, 724 (2009).

${ }^{24}$ E. Gavartin, R. Braive, I. Sagnes, O. Arcizet, A. Beveratos, T. J. Kippenberg, and I. Robert-Philip, Phys. Rev. Lett. 106, 203902 (2011).

${ }^{25}$ P. Kaer, T. R. Nielsen, P. Lodahl, A.-P. Jauho, and J. Mørk, Phys. Rev. B 86, 085302 (2012).

${ }^{26}$ L. Sapienza, H. Thyrrestrup, S. Stobbe, P. D. Garcia, S. Smolka, and P. Lodahl, Science 327, 1352 (2010).

${ }^{27}$ H. J. Carmichael, R. J. Brecha, M. G. Raizen, H. J. Kimble, and P. R. Rice, Phys. Rev. A 40, 5516 (1989).

${ }^{28}$ A. J. Ramsay, T. M. Godden, S. J. Boyle, E. M. Gauger, A. Nazir, B. W. Lovett, A. M. Fox, and M. S. Skolnick, Phys. Rev. Lett. 105, 177402 (2010)

${ }^{29}$ B. Krummheuer, V. M. Axt, and T. Kuhn, Phys. Rev. B 65, 195313 (2002).

${ }^{30}$ J. Johansen, S. Stobbe, I. S. Nikolaev, T. Lund-Hansen, P. T. Kristensen, J. M. Hvam, W. L. Vos, and P. Lodahl, Phys. Rev. B 77, 073303 (2008).

${ }^{31}$ S. Stobbe, J. Johansen, P. T. Kristensen, J. M. Hvam, and P. Lodahl, Phys. Rev. B 80, 155307 (2009).

${ }^{32}$ S. Laurent, S. Varoutsis, L. Le Gratiet, A. Lemaître, I. Sagnes, F. Raineri, A. Levenson, I. Robert-Philip, and I. Abram, Appl. Phys. Lett. 87, 163107 (2005). 